\title{
Efectos del sedentarismo y obesidad en el desarrollo psicomotor en niños y niñas: Una revisión de la actualidad latinoamericana
}

\author{
Effects of physical inactivity and obesity in psychomotor development in children: A review of Latin American \\ news
}

Igor Cigarroa', Carla Sarqui², Rafael Zapata-Lamana ${ }^{3}$

1 Licenciado en Kinesiología. Magíster en Neurorehabilitación, Máster en Neurociencias, Carrera de Kinesiología, Facultad de Salud, Universidad Santo Tomas. Los Ángeles, Chile. Investigador del Institut de Neurociències, Departament de Psiquiatria i Medicina Legal, Universitat Autònoma de Barcelona. Barcelona, España. e-mail: icigarroa@santotomas.cl

2 Licenciada en Kinesiología, Carrera de Kinesiología. Facultad de Salud, Universidad Santo Tomás. Los Ángeles, Chile. email: carla.sarqui@alumnos.santotomas.cl

3 Profesor de Educación Física. Magíster en Medicina y Ciencias del Deporte, Escuela de Educación, Universidad de Concepción. Los Ángeles, Chile. e-mail: zapatalamanar@gmail.com

Cigarroa I, Sarqui C, Zapata-Lamana R. Efectos del sedentarismo y obesidad en el desarrollo psicomotor en niños y niñas: Una revisión de la actualidad latinoamericana. Univ. Salud. 2016;18(1):156-169. DOI: http://dx.doi.org/10.22267/rus.161801.27

\begin{abstract}
Resumen
Introducción: En 1995 la Organización Mundial de la Salud declaró la obesidad como una enfermedad. Actualmente, se considera una epidemia en adultos y niños. Su presencia en edades tempranas es factor de riesgo de obesidad y riesgo coronario en la adultez. Los niños con sobrepeso y obesidad practican menos actividad física y más actividades sedentarias. Estos estilos de vida son tal vez los principales responsables de los limitados patrones motores que presentan. Objetivo: Realizar una revisión y compilación de los estudios actuales en Latinoamérica que hablen sobre la relación entre obesidad, sedentarismo y desarrollo psicomotor infantil. Materiales y métodos: Se realizó la búsqueda en Pubmed y Scielo. Las palabras clave utilizadas fueron: desarrollo psicomotor, desarrollo motor, retraso en el desarrollo psicomotor, sobrepeso infantil, obesidad infantil, nutrición infantil. Resultados: Existe evidencia que apoya la relación entre sobrepeso/obesidad infantil y un bajo rendimiento en pruebas psicomotoras en estudios realizados en Latinoamérica. No se encontraron estudios de establezcan una relación positiva entre sobrepeso y obesidad y mejores puntajes en test psicomotrices. Conclusiones: Los resultados confirman el negativo impacto que tiene la malnutrición por exceso, en el desarrollo psicomotriz de niños y niñas. Aun cuando, una mayor cantidad de estudios experimentales son necesarios para confirmarlo.
\end{abstract}

Palabras clave: Sobrepeso; obesidad; estilo de vida sedentario; destreza motora. (Fuente: DeCS, Bireme).

\begin{abstract}
Introduction: In 1995 the World Health Organization declared obesity as a disease. Currently, obesity is considered an epidemic in both adults and children. The presence of this disease at an early age is a risk factor for obesity and heart disease in adulthood. Increasingly, overweight and obese children practice less physical activity and they are more interested in sedentary activities. These lifestyles are perhaps the main cause of low performance in motor patterns. Objective: To conduct a review and compilation of current studies in Latin America to talk about the relationship between obesity, sedentary lifestyle and children's psychomotor development. Materials and methods:
\end{abstract}


The search was conducted in Pubmed and Scielo. The keywords used were: psychomotor, motor development, delayed motor development, childhood overweight, childhood obesity and childhood nutrition. Results: There is evidence supporting the relationship between childhood overweight/obesity with poor performance in psychomotor tests in Latin America studies. No studies that establish a positive relationship between overweight and obesity with better psychomotor test scores were found. Conclusions: The results confirm the negative impact of malnutrition due to excess of food in children's psychomotor development. Although a greater number of experimental studies are needed to confirm this statement.

Keywords: Overweight; obesity; sedentary lifestyle; motor skills. (Source: DeCS, Bireme).

\section{Introducción}

En la etapa de crecimiento los niños encuentran en su cuerpo y el movimiento las principales vías para iniciar el contacto con el entorno y, de esta forma, obtener los primeros conocimientos acerca del mundo en el que están creciendo y desarrollándose.1,2 El progresivo descubrimiento de su propio cuerpo como fuente de sensaciones, exploración de posibilidades de acción y funciones corporales, constituirán experiencias imprescindibles sobre las que se irá desplegando un correcto desarrollo psicomotor.1-3 Para cada niño el descubrimiento del entorno conlleva el aprendizaje de un conjunto de habilidades cada vez más complejas que llamaremos desarrollo psicomotor. El aprendizaje de estas habilidades las logrará a través de la práctica continua que lo llevará a cambio permanente de sus capacidades físicas motoras, cognitivas, sociales $\mathrm{y}$ emocionales.1,3

Existen una gran cantidad de factores propios de cada individuo, como ambientales que se presentan a lo largo del desarrollo de los niños que pueden animar o desanimar la exploración y el movimiento natural y espontaneo de los niños.1 El desarrollo de las habilidades psicomotoras, desde la perspectiva de una práctica adecuada al nivel de desarrollo infantil, debería plantearse como una prioridad en los programas de educación física. Lamentablemente, en los últimos tiempos niños y adolescentes, vienen practicando menos actividad física convirtiéndose esta inactividad en una de las grandes causas del aumento del peso corporal, y de la baja competencia motriz en los niños.4,5

La obesidad es considerada en la actualidad una epidemia mundial, suele iniciarse en la infancia y se considera una enfermedad crónica, compleja y multifactorial que genera alteraciones funcionales, de composición bioquímica, y de estructura corporal, obviando las causas genéticas, existen variables de tipo cognitivo, afectivas y conductuales que la provocan.5 El bajo tiempo dedicado a la actividad física, el exagerado tiempo dedicado a las actividades sedentarias o los malos hábitos alimentarios son conductas que se están adoptando en gran parte de la población infantil occidental.1,2,6-8 Ejemplo de esto son el uso indiscriminado de pantallas (celulares, portátiles, ordenadores, juegos de video) que repercute directamente en la menor cantidad de horas de actividad física en los niños o el consumo abusivo de azúcares y grasas saturadas y no de comidas "saludables" que repercute directamente en la alta tasa de obesidad infantil que tenemos en estos días. 6,9,8,10,11

En general, los niños obesos y con sobrepeso se cansan más rápido, ejecutan y presentan una competencia motriz inferior a la esperada para su edad, en el equilibrio, carrera, carrera lateral, galopar, saltos, recibir, lanzar, rebatir, chutar y golpear un balón.12 Los niveles inferiores de aptitud física conlleva a que interactúen menos con su entorno, disminuyan la cantidad de actividad física, y posteriormente termine por excluir a los niños de diversas actividades físicas y bromas infantiles, conduciéndolos a la inactividad y al aislamiento en los momentos de juego.1,2,13 Las bajas oportunidades de movimiento tienen un impacto negativo en su desarrollo conduciéndoles a niveles de habilidades motoras muy pobres.

Estos fenómenos, nos llevan a hacer pensar que los niños con sobrepeso y obesidad presentar menores habilidades en el desarrollo psicomotor 
y puntuaciones más bajas en test estandarizados de desarrollo psicomotor en comparación con los niños con diagnóstico nutricional de peso normal. No se han encontrado una gran cantidad de literatura de investigaciones realizadas en Latinoamérica que hablen sobre sedentarismo, obesidad y su relación con en el desarrollo motor normal de los niños. Este artículo tiene como objetivo hacer una revisión y compilación de los estudios actuales en Latinoamérica que hablen sobre la relación entre obesidad, sedentarismo y desarrollo psicomotor infantil. De esta forma, determinar si realmente se ha encontrado una asociación entre estas variables y si la presencia de un diagnóstico de malnutrición por exceso se asocia directamente a un diagnóstico de déficit del desarrollo psicomotor. Un mejor entendimiento de la relación entre estas variables permitirá clarificar la relación y posibles efectos del sedentarismo y obesidad infantil en el desarrollo psicomotor infantil.

\section{Materiales y métodos}

El presente estudio es un artículo de revisión que buscó en la literatura estudios actuales que establezcan la relación existente entre sobrepeso/obesidad y desarrollo motor en niños de ambos sexos, los motores de búsqueda fueron Pubmed y Scielo. La búsqueda se realizó en castellano e inglés, se concentró en investigaciones en humanos hechas en Latinoamérica de los últimos 10 años (del 2005 al 2015). Las palabras de búsqueda en castellano fueron: desarrollo psicomotor, desarrollo psicomotriz, desarrollo motor, coordinación motora, crecimiento infantil, conducta infantil, sobrepeso infantil, obesidad infantil, nutrición infantil. Las palabras de búsqueda en ingles fueron: psychomotor development, psychomotor, development, motor skills, child behavior, childish behavior infant behavior, childhood overweight, childhood obesity, child nutrition.

\section{Resultados}

Desarrollo psicomotor: El desarrollo psicomotor se define como el conjunto de habilidades que el niño va adquiriendo desde que nace y durante los dos primeros años de vida es principalmente intenso ${ }_{1}$, lo componen el sistema sensorial, motor, mental y una interacción entre el lenguaje y el área social del individuo, es un proceso de continuo cambio, en el que el niño comienza a dominar niveles cada vez más complejos de movimiento, pensamiento, relaciones con los demás, con los objetos y el medio ambiente. 1,14

Cada ser humano al nacer tiene un potencial de desarrollo determinado congénitamente. El resultado final será la combinación entre la carga genética y los estímulos recibidos por parte de su entorno familiar, social y con el medio ambiente. $\mathrm{Si}$ consideramos a la carga genética como la herencia que nos traspasan nuestros padres sobre la cual, no tenemos decisión y posibilidad de cambio, la opción que nos queda es modificar a nuestro favor el ambiente y la cantidad y calidad de estímulos a los que nos vemos expuestos. En este sentido, una estimulación temprana y efectiva tendría un impacto positivo en la generación de un desarrollo psicomotor normal y en la corrección de un posible déficit.1,2 El niño está en continua evolución, y a medida que transcurren los años, las experiencias vividas terminan por pulir los patrones motores con los que nace.2 El movimiento del niño se desarrolla y mejora produciéndose modificaciones cuantitativas expresadas en el crecimiento físico; aumentando la estatura y peso corporal, y de orden cualitativo, como la adquisición de las funciones motoras.2

Aprendizaje motor: El aprendizaje está basado en la práctica, Schmidt definió aprendizaje motor como los procesos internos asociados con la práctica que provocan cambios relativamente permanentes en la capacidad motriz, los cuales surgen en el transcurso de una estimulación adecuada y suficiente para una mejora relativa y permanente en el desempeño de las habilidades motoras.1,2,3 Desde las acciones más simples que realizan los niños hasta las más complejas de los deportistas de elite, todas son resultado de un proceso progresivo de adquisición de habilidades.1,3 El aprendizaje motor no es un proceso que avanza de manera irregular $y$ 
azarosa, sino que ocurre de manera ordenada y lineal siguiendo una serie de fases o estadios que podrían ser identificados como comunes a todos los individuos. Así se pone de relieve que todo nuevo aprendizaje se organiza de manera secuenciada y progresiva, integrando las estructuras aprendidas a las nuevas estructuras.1

Desde su nacimiento, el niño mediante el movimiento descubre conoce y controla cuerpo, lo que le permite más tarde actuar en forma autónoma y libre con iniciativa, seguridad y confianza en sí mismo.1 Los reflejos son los primeros movimientos que los niños realizan al nacer, manteniéndose algunos de estos durante toda la vida, los cuales tienen una importancia fundamental en las adquisiciones motrices futuras, dado que son la base de la constitución de toda la motricidad del ser humano, en este periodo también se distinguen los movimientos estereotipados como patalear, balancearse, entre otros.1

A medida que el niño crece comienza a realizar movimientos voluntarios muy primitivos $y$ pobremente integrados en un principio, esta etapa se caracteriza en que los niños comienzan a tomar conciencia y a reconocer partes de su propio cuerpo.1 Los movimientos voluntarios de manipulación son combinados con los movimientos visuales y táctiles luego pasan por diferentes fases hasta llegar a la adquisición de movimientos manipulativos fundamentales altamente coordinados y especializados, como lanzar, atacar, coger, y la adquisición de habilidades especiales, como botar una pelota, saltar la cuerda, entre otros. A medida que el niño se desarrolla y practica las habilidades aprendidas, va adquiriendo mayor control y precisión de estos movimientos hasta llegar al dominio y perfección de los movimientos básicos fundamentales como saltar, correr, trepar, lanzar, entre otros.1,2 Los patrones básicos de movimiento van desarrollándose en forma secuencial, lo cual permite distinguir en diferentes etapas o hitos de aprendizaje, hasta llegar a la habilidad madura del movimiento.1

Sedentarismo infantil: Aunque parezca contraintuitivo la mayor reducción de actividad física a lo largo de la vida no se produce en adultez, sino que empieza mucho tiempo antes, específicamente en el periodo de la infancia y preadolescencia ¿Qué causa el sedentarismo? Existen diferentes factores que pudieran explicar por qué el descenso de la actividad física se intensifica en este periodo vital de desarrollo y movimiento. Algunos de estos factores son básicamente biológicos e inherentes a la fisiología del niño, mientras que otros factores tienen un origen socio-cultural, resultado de la situación social y económica actual de los países.

\section{Factores fisiológicos}

El propio desarrollo fisiológico del niño tiende, de forma natural, a provocar una disminución de la actividad física, dedicándole más tiempo y atención a actividades de orden más intelectual (exámenes, test de selectividad, rendimiento escolar). En el periodo previo a la pubertad, el niño se vuelve más sedentario y su nivel de actividad física disminuye en relación a etapas vitales anteriores. 15

\section{Factores socio-culturales}

Desafortunadamente, esta disminución de la actividad física se ve agravada por otros factores socio-culturales como: los cambios en los tipos de juegos, en los hábitos alimentarios y la presión por rendimiento académico.

\section{¿Qué pasó con la forma de diversión y pasar los tiempos de ocio de los niños?}

Los juegos y formas de diversión han cambiado drásticamente en los últimos años. A principios del siglo XX se jugaba principalmente con los recursos propios del campo, de la plaza o del patio. Los juegos normalmente son enraizados con oficios, los juguetes tradicionales como los caballos de madera, polcas, muñecas, la cuerda, eran fabricados de forma artesanal.14 Los niños actualmente, son capaces de mantenerse por horas sentados frente a una pantalla (televisión, computador, dispositivos móviles, tabletas, etc.).7,9,14 Y su uso o mejor dicho abuso se ha asociado a una menor participación en actividades de tipo físico-deportivo y patologías crónicas que antiguamente se asociaban casi exclusivamente a edades adultas. Además, recientes investigaciones asocian jugar muchas 
horas en consolas de videojuego con problemas de atención y concentración.6,16

Otro factor socio-cultural que ha contribuido a la disminución de actividad física en niños está relacionado con el aumento de la presión y al rendimiento académico ya en edades muy tempranas. En muchos casos, la necesidad de aumentar el rendimiento académico provoca la sustitución de clases de actividad física escolares o actividades deportivas extra-curriculares por actividades con contenidos puramente académicos.17, 18 Un reporte del Instituto de investigación social de la Universidad de Michigan indica que algunos colegios en Estados Unidos han reducido las oportunidades para jugar en un $25 \%$ y las actividades al aire libre en un 50\%.19,20

Según datos de la Organización Mundial de la Salud (OMS), la inactividad física representa el 6\% de las defunciones a nivel mundial, ocupando el $4^{\circ}$ lugar antecedido por la hipertensión (13\%), consumo de tabaco (9\%), y el exceso de glucosa en la sangre (6\%). Esta condición representa la principal causa de aproximadamente el $21-25 \%$ de los cánceres de mama y de colon, $27 \%$ de la diabetes y un $30 \%$ de las cardiopatías isquémicas.

Como forma de combatir este explosivo aumento de enfermedades crónicas no trasmisibles (ECNT). La OMS lanzó el 2010 las recomendaciones mundiales sobre actividad física para la salud. En el grupo de 5 a 15 años recomienda al menos 60 minutos diarios de actividad física moderada o vigorosa. Pues se ha visto que práctica diaria de ejercicio está fuertemente asociada a parámetros de salud favorables, mejora las funciones cardiorrespiratorias y de la fuerza muscular, reduce la grasa corporal, ayuda a prevenir las enfermedades cardiovasculares y metabólicas, mejora la salud ósea y disminuye la presencia de síntomas de depresión.21
Si al sedentarismo le agregamos además, un alto consumo de alimentos de alta densidad energética y bajo valor nutricional y de bebidas con azúcar de alta palatabilidad que no producen saciedad en el niño, obtenemos como resultado un aumento explosivo de las tasas de obesidad infantil.10,11,22

Obesidad infantil: El año 1995 la OMS declaró a la obesidad como una enfermedad y sumado al sobrepeso constituyen el $5 \%$ de la mortalidad mundial.21 En la actualidad, la obesidad se considera una epidemia en los países occidentalizados, tanto en adultos y adolescentes como en niños. Además, no solo es distinguida por el aumento de peso corporal, (exceso de grasa acumulada en el tejido adiposo y menor cantidad de tejido muscular y masa ósea) sino como una enfermedad metabólica e inflamatoria, de curso crónico, multifactorial y de gran repercusión en la salud pública, por las consecuencias biológicas, psicosociales y económicas que tiene en la población.23 Las causas del sobrepeso y la obesidad en niños están relacionadas a factores genéticos y estilos de vida.24 Mientras que las modificaciones genéticas de un individuo no son posibles, algunas de las causas conductuales relacionadas a los estilos de vida si lo son, particularmente con lo que tiene que ver con los hábitos de ejercicio y nutrición. La obesidad infantil se asocia al alto consumo de alimentos ricos en energía derivada de los carbohidratos, en grasas saturadas y sal, acompañados de falta de actividad física (sedentarismo) e incremento de actividades sedentarias asociadas a los actuales estilos de vida de gran parte de la población infantil (uso de videojuegos pasivos, internet, televisión, teléfono celular, etc.) El modelo de promoción de salud HPM (de sus siglas en inglés) establece las variables conductuales, afectivas, cognitivas y demográficas que más podrían estar relacionadas con el sobrepeso y la obesidad. (Figura 1). 
Figura 1. Causas, evaluación y consecuencias físicas y psicológicas de la obesidad infantil

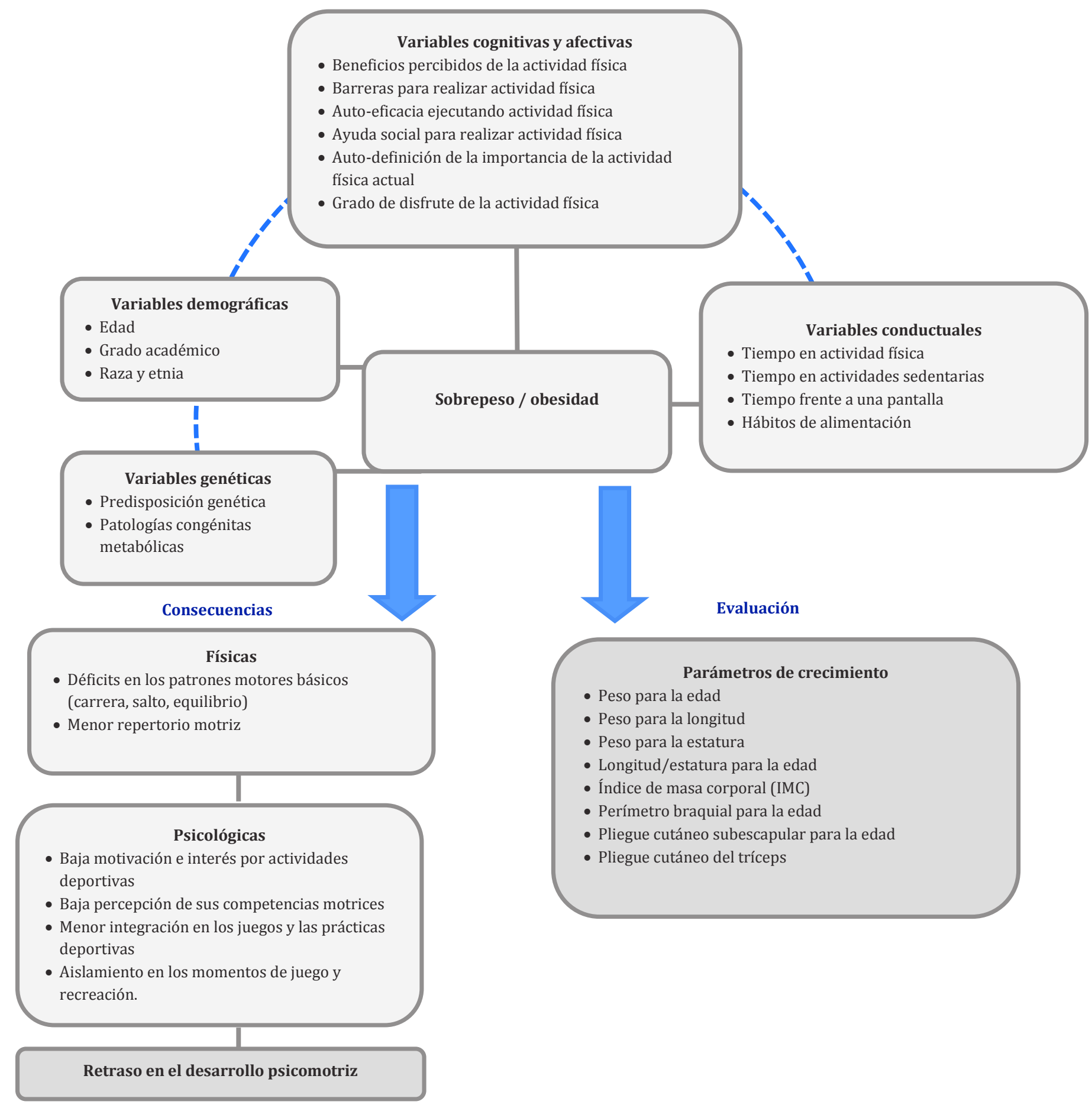


Utilizando como referencia estas variables Vanden et al., en el 2014 realizaron un estudio a 73 niñas de $6^{\circ}$ y $7^{\circ}$ grado, estableciendo su relación con el Índice de Masa Corporal (IMC).25 Las variables que más se relacionaron con el aumento del IMC fueron: los obstáculos para realizar actividad física, los beneficios que se percibe se obtienen de la actividad física, el tiempo en el día que se le dedica a practicar distintos tipos de actividad física y el tiempo que se le dedica a las actividades de sedentarismo. Además, el estudio mostró que al comparar los grupos normopeso y sobrepeso/obesidad se encontraron diferencias en las variable cognitiva y afectiva: disfrute de la actividad física. Las niñas de peso normal presentaban mayor disfrute de la actividad física que las niñas obesas. En relación a las variables conductuales, la única diferencia significativa entre las niñas obesas y no obesas, fue la realización de actividad física vigorosa. Las niñas obesas practican en menor cantidad y además ocupaban más tiempo en actividades sedentarias. Concluyendo que el IMC se correlacionaba significativamente con las variables cognitivas, afectivas y variables de comportamiento, pero no con las variables demográficas.

En Chile, la obesidad infantil es un problema de salud creciente al igual que en todo el mundo.26,27 En el pasado y actualmente, en este país se han implementado estrategias para mejorar los índices de salud de la población. A modo de resumen, se hizo el lanzamiento de un programa interministerial llamado "Elige Vivir Sano", se establecieron metas sanitarias para el periodo 2011-2020, se incorporó dentro del Sistema de Medición de la Calidad de la Educación (SIMCE) un apartado para la medición de las habilidades físicas de los niños, se re-estructuró la asignatura de Educación Física por parte del Ministerio de Educación, (MINEDUC) dándole una orientación hacia las mejoras en la salud a través de estilos de vida más sanos, se creó un Ministerio del Deporte y actualmente, este ministerio, junto con el de Educación y Salud han conformado una mesa intersectorial de expertos para elaborar las recomendaciones de actividad física para la población chilena para los próximos años. Pese a ello, las estadísticas de salud poblacional no han mejorado sustancialmente. La prevalencia de sobrepeso y obesidad en menores de seis años ha crecido en los últimos años, se estima que desde el 2005 al 2011 ha incrementado de un 51,4\% especialmente, a expensas del sobrepeso.28 Independiente de la encuesta, Chile tiene porcentajes de malnutrición por exceso en los menores de seis años superiores al 30\%, de los cuales aproximadamente un $10 \%$ son obesos.29,30

Según el Informe de Salud del 2011 de la Organización para la Cooperación y el Desarrollo Económico (OCDE), Chile se encuentra en el $4^{\circ}$ puesto de los países miembros con mayor cantidad de obesidad adulta y el $6^{0}$ con mayor sobrepeso infantil.31 En otros países de Sudamérica, la realidad no es muy diferente. Un estudio realizado en el 2011 en tres escuelas de Buenos Aires en 534 niños de entre 5 y 10 años, concluyó que existe un alto porcentaje de niños con exceso de peso obteniendo el binomio sobrepeso obesidad de un $47,4 \%$ de los cuales el $20,4 \%$ se encuentra en estado de obesidad. 32 Por otro lado, según datos del Ministerio de Salud de Perú, en el 2008 los niños menores de cinco años con sobrepeso y obesidad fueron de $4,8 \%$, cifra en alza para el año 2010, llegando a un 6\%. En los niños de cinco a nueve años, en el 2008 el sobrepeso fue de $7,8 \%$.

La presencia de obesidad en edades tempranas es un factor de riesgo para la obesidad y riesgo coronario en la edad adulta, además, se asocia a varios factores de riesgo de enfermedades cardiovasculares (presión sistólica y diastólica aumentada, elevados niveles de lípidos en sangre, colesterol total y triglicéridos) de ahí la importancia de prevenirla, detectarla y tratarla oportunamente.33

Para hacer el diagnóstico de sobrepeso y obesidad es necesario contar con indicadores antropométricos que se asocien con la adiposidad. El índice de masa corporal (IMC) ha resultado útil para la evaluación en el adulto, y en tiempos recientes se ha recomendado para la evaluación de niños y adolescentes. Además del IMC, en el caso de los niños, existen otras mediciones antropométricas para valorar la talla 
(crecimiento) y su composición corporal. Entre ellas se incluyen:

1. Peso para la edad : En ambos sexos, en edades comprendidas desde el nacimiento a los seis meses; nacimiento a los dos años; seis meses a dos años; dos a cinco años y desde el nacimiento a los cinco años.

2. Peso para la longitud: En ambos sexos, desde el nacimiento a los dos años.

3. Peso para la estatura: En ambos sexos, de los dos a los cinco años.

4. Longitud/estatura para la edad: En ambos sexos, en edades comprendidas desde el nacimiento a los seis meses; nacimiento a los dos años; seis meses a dos años; dos a cinco años y desde el nacimiento hasta los cinco años.

5. IMC para la edad: Un indicador simple de la relación entre el peso y la talla, utilizado frecuentemente para identificar el sobrepeso y la obesidad. Para ambos sexos, comprende edades desde el nacimiento hasta los dos años; de dos a cinco años y desde el nacimiento hasta cinco años.

6. Perímetro braquial para la edad: Las ventajas que tiene este tipo de medición, es que guarda buena correlación con el índice peso edad, además de sugerir disponibilidad de reservas en forma de grasa subcutánea. Las tablas entregadas por la OMS, comprenden las edades de tres meses a cinco años, para ambos sexos.

7. Pliegue cutáneo subescapular para la edad: Esta medición, valora la cantidad de tejido adiposo subcutáneo. Las tablas entregadas por la OMS, comprenden edades de tres meses a cinco años, en ambos sexos.

8. Pliegue cutáneo del tríceps: Valora la cantidad de tejido adiposo subcutáneo. Las tablas comprenden edades de tres meses a cinco años, en ambos sexos.

Todos los resultados de las mediciones antropométricas enunciadas son expresados en puntaje z y percentiles.

Es fundamental obtener estas mediciones con la técnica y el instrumental adecuados. Una vez tomados las medidas, para interpretarlas, es necesario contrastarlas con las de sus familiares y con los patrones de referencia, que se puede hacer mediante percentiles o calculando puntuaciones Z. Tanto la OMS, la CDC, como estudios locales realizados en distintos países, han establecido dependiendo de la edad y género, patrones de crecimiento poblacionales que sirven de referencia para detectar individuos y/o grupos de riesgo nutricional. Así, tenemos una norma que nos permite saber el estado de salud de una población. En la tabla 1 se muestran los índices nutricionales para niños de cero a cinco años.

Tabla 1. Índices nutricionales derivados del peso, de la talla y del IMC

\begin{tabular}{|c|c|c|}
\hline $\begin{array}{c}\text { Relación o } \\
\text { índice }\end{array}$ & Cálculo & Clasificación \\
\hline \multirow[t]{2}{*}{$\begin{array}{l}\text { Relación } \\
\text { peso/talla }\end{array}$} & $\begin{array}{l}\text { Curva de } \\
\text { percentiles / } \\
\text { puntuación z }\end{array}$ & $\begin{array}{l}\text { Relación peso/talla.34* } \\
\text { - Normal: P15- P85 ( } \mathrm{z} \geq-1 \mathrm{y} \leq \\
+1 \text { ) }\end{array}$ \\
\hline & & $\begin{array}{l}\text { - Subnutrición (tres niveles) } \\
\text { a) Leve, P3 }(z<-1 y \geq-2) \\
\text { b) Moderada, } z<2 \geq-3 \\
\text { c) Grave, } z<-3 \\
\text { - Sobrenutrición (tres niveles) } \\
\text { a) Leve (sobrepeso) }>\text { P85 y }< \\
\text { P97 ( }>+1 \text { y } \leq+2) \\
\text { b) Obesidad, }>\text { P97 ( } z>+2 y \leq+3) \\
\text { c) Obesidad intensa, } z>+3\end{array}$ \\
\hline \multirow{4}{*}{$\begin{array}{l}\text { Índice de } \\
\text { Masa } \\
\text { Corporal } \\
\text { (IMC) }\end{array}$} & $\begin{array}{l}\text { Peso }(\mathrm{kg}) / \\
\text { talla }(\mathrm{m})^{2}\end{array}$ & $\begin{array}{l}\text { IMC. } 35^{* *} \\
\text { - Normal: P15- P85 ( } \mathrm{z} \geq-1 \mathrm{y} \leq \leq \\
+1)\end{array}$ \\
\hline & & $\begin{array}{l}\text { - Sobrepeso: > P85 (puntuación } \\
\mathrm{z}>+1 \text { ), equivalente a un IMC de } \\
25 \mathrm{~kg} / \mathrm{m} 2 \text { a los } 19 \text { años.*** }\end{array}$ \\
\hline & & $\begin{array}{l}\text { - Obesidad: > P98 (puntuación } \\
\text { z > +2), equivalente a un IMC de } \\
30 \mathrm{~kg} / \mathrm{m} 2 \text { a los } 19 \text { años. }{ }^{* *}\end{array}$ \\
\hline & & - Subnutrición: < P3 $(\mathrm{z}<-2)$ \\
\hline
\end{tabular}

Si los niños presentan obesidad en etapas tempranas del desarrollo (dos a seis años) es muy probable que se mantengan en esta condición nutricional en las siguientes fases del desarrollo (seis a diez años), lo que perjudicará su crecimiento y desarrollo normal. 
La obesidad limita el desarrollo en forma integral, los niños con sobrepeso y obesidad que presentan una baja percepción de competencia motriz en los momentos de juego, no se verán lo suficientemente capaces de realizar muchas de las actividades que sus pares realizan sin dificultad, lo cual llevará a una menor motivación e integración a las prácticas deportivas y recreativas con sus compañeros y condicionará al aislamiento y al rechazo en los momentos de juego.2

Actualmente, los niños se interesan y practican menos actividad física, esta característica se ve mucho más acentuada en los niños con sobrepeso y obesidad.2 Se ha visto que el desempeño motor se mejora con la práctica de actividad física, donde la ejecución motora de un niño esta correlacionada con la cantidad y diversidad de propuestas motrices que se le ofrecen.2

Diversas estrategias, tanto a corto, como largo plazo, han sido planteadas en escuelas y jardines infantiles para el manejo de la obesidad infantil, las cuales han sido dirigidas principalmente a la modificación de la ingesta, siendo escasas las intervenciones que promueven aumentar la actividad física, educar y capacitar a los profesores y padres, tanto a nivel preventivo, como terapéutico.36-40 Establecer en los primeros años de vida el hábito de actividad física tiene un efecto positivo en la niñez; otorgando un óptimo desarrollo y crecimiento, como también en edades posteriores, al prevenir enfermedades crónicas y por sobre todo entregando los pilares de un estilo de vida activo y saludable.41 Un estudio realizado por Carrasco, 42 demostró que la alimentación y el ejercicio físico por sí solo, no producían una reducción significativa del peso en las personas, sin embargo, en quienes realizaban dieta combinada con el ejercicio lograban una mayor disminución del peso corporal.

Obesidad y alteraciones en el desarrollo psicomotor: Los estudios centrados en relacionar las consecuencias de la obesidad y alteraciones en el desarrollo psicomotor son escasos y contrapuestos a la cantidad de estudios que relacionan la variable desarrollo psicomotor con desnutrición.43 Pese a ello, existen estudios en
Latinoamérica que hablan sobre la asociación entre bajos puntajes en test de desarrollo psicomotor o motor infantil y sobrepeso/obesidad. Bucco en el 2012 analizó la evolución de la edad cronológica con la edad motora general de niños con sobrepeso/obesidad.

Tomó los percentiles del IMC utilizando el patrón del Sistema de Evaluación del Estado Nutricional Infantil según el NCHS y la Escala de Desarrollo Motor desarrollada por Francisco Rosa Neto en 284 niños entre seis y diez años. Los resultados indicaron que los niños con sobrepeso/obesidad presentaron un desarrollo motor inferior en relación a la edad cronológica. Los niños mostraron una deficiencia estadísticamente significativa $(p \leq 0,005)$ en todas las estructuras motoras. Específicamente, mostraron un retraso en el equilibrio $(p \leq 0,001)$, esquema corporal $(\mathrm{p} \leq 0,0029)$ y organización espacial $(\mathrm{p} \leq 0,001)$. Por esto, se concluyó que los niños con sobre peso/obesidad tienen un retraso con respecto a sus compañeros de la misma edad de peso normal; este resultado se atribuyó a las características físicas de los niños y a sus estilos de vida no saludables.5

En otro estudio realizado por Bucco en el 2013, mostró que existían diferencias motoras entre niños y niñas de 6 a 10 años con sobrepeso y obesidad versus normopesos de la misma edad, concluyendo que las niñas presentan niveles de desempeño inferiores que los niños. Este resultado lo atribuyó al estilo de juego que practicaba habitualmente cada grupo. En relación a las áreas específicas de ejecución motora, identificó un retraso motor mayor en los niños de ambos sexos con obesidad y sobrepeso en las áreas de equilibrio, organización temporal, organización espacial y esquema corporal.2

Por otra parte, un estudio realizado en Perú a 4.007 niños con edades entre los 6 y 11 años, mostró que el nivel de adiposidad presentaba una influencia significativa en los resultados de cada una de las pruebas de coordinación. Los niños con adiposidad elevada tenían rendimientos inferiores en comparación con los de adiposidad reducida en dos de las cuatro pruebas (saltos 
monopedales y la marcha hacia la retaguardia). Además, observó un desarrollo coordinativo inferior en los niños de adiposidad elevada en comparación con los que presentan adiposidad reducida.44 Resultados muy similares fueron encontrados en un estudio publicado por Slining en el 2010 en el "Journal of pediatric", quien mostró que existía un retraso motor de 1,8 veces más probable en los niños menores de 3 a 18 meses con sobrepeso en comparación a los niños normopeso. Además, se encontró que los lactantes con alto contenido de grasa subcutánea tenían 2,32\% veces más probabilidad de presentar retraso motor en comparación con niños con menos grasa subcutánea. 43

Son variadas las escalas en las cuales se ha encontrado la relación entre un diagnóstico de obesidad y déficit en el desarrollo psicomotriz. Por ejemplo, en un estudio realizado en Chile en 23 preescolares de cinco años en el 2014, relacionó el estado nutricional con puntaje z-IMC, el desarrollo psicomotor con la batería $\mathrm{Da}$ Fonseca y la cantidad e intensidad de actividad física con actigrafía en la jornada escolar en preescolares de cinco años. Los resultados arrojaron que el $78 \%$ de los niños se encontraba entre sobrepeso y obesidad. Todos los niños realizaron al menos 60 minutos de actividad física moderada a vigorosa durante la jornada escolar y el 17,4\% estaba en la categoría de dispraxia.

Se encontró una relación inversa significativa entre z-IMC y el desarrollo psicomotriz, por lo cual se concluyó que los niños con mayor z-IMC tenían menor puntaje en la batería Da Fonseca.45 En otro estudio de Méndez Ruiz M. en 2015, se comparó la distribución del desarrollo psicomotor en tres diagnósticos nutricionales: normal, sobrepeso y obeso.

Para ello se aplicó el Test de DSM "TEPSI" a 58 niños, de entre cuatro y cuatro y medio años. Los preescolares con obesidad y sobrepeso estudiados mostraron un perfil motriz clasificado como inferior y muy inferior, respectivamente, cuando eran comparados con el grupo de preescolares normopeso, que permitió concluir que el sobrepeso/obesidad es un factor que influye en el desarrollo de la psicomotricidad de forma negativa en preescolares de cuatro años de

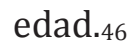

No se encontraron artículos que hablaran sobre efectos positivos de la obesidad en el desarrollo psicomotriz, o sobre una relación positiva entre el diagnóstico de sobrepeso/obesidad y desarrollo psicomotriz. Sólo se encontró un estudio realizado en Colombia en el 2013 que evaluó a 800 escolares de entre de 8 a 12 años. A los menores con un IMC> al percentil 85 se les aplicó una encuesta sociodemográfica, el cuestionario de actividad física del INTA, el test de marcha de seis minutos y la batería de evaluación psicomotriz de Da Fonseca. Pese a que los niños con obesidad presentaban ejecuciones limítrofes en la batería, no se evidenció alteraciones de perfil psicomotor en los niños y niñas con sobrepeso y obesidad. 47

\section{Discusión}

Si bien se pudo encontrar evidencia en la literatura latinoamericana que hable sobre los efectos negativos del sobrepeso y la obesidad infantil a nivel físico y psicológico, existe poca evidencia que establezca una relación entre diagnósticos nutricionales de sobrepeso y obesidad con bajos rendimientos en pruebas psicomotoras y motoras.

Los cambios en los hábitos de actividad física en los niños y las presiones académicas por obtener mayor rendimiento se han planteado como los principales responsables del sedentarismo infantil. Sumado a los cambios en los hábitos alimentarios, algunas características conductuales, emotivas y cognitivas detectadas en los niños sedentarios han llevado a aumentar considerablemente los niveles sobrepeso/obesidad infantil en la actualidad.

La pobre interacción con medios ambientes naturales y la restricción de movimiento desde edades cada vez más tempranas, conlleva a una pobre ejecución de propuestas motrices necesarias para un correcto desarrollo psicomotor. Como resultado, estos niños no presentan las competencias motrices necesarias 
durante los momentos de juego, esto los llevará a ser rechazados en los momentos de juego y a ocupar los tiempos libres en actividades que promueven al sedentarismo y aún más a la obesidad.

La evidencia disponible sugiere que niños de ambos sexos clasificados como sobrepesos $\mathrm{u}$ obesos, presentan alteraciones en su desarrollo psicomotor en diversas áreas, según los resultados arrojados por diversos instrumentos de medición del desarrollo motor. Sin embargo, se pueden apreciar algunas diferencias en el rendimiento motriz según género. Se observa una tendencia en las niñas a presentar un bajo rendimiento motriz en habilidades que exigen la presencia de patrones motores básicos (carrera, salto, equilibrio) y un mejor rendimiento en actividad de motricidad fina en relación a los niños.

Las causas se podrían atribuir a una menor práctica en la primera situación y una mayor práctica en el segundo tipo de propuestas motrices, como resultado del tipo de juego que estas prefieren y juegan. Bajo este contexto, los ambientes educacionales como: salas cuna, jardines infantiles y escuelas constituyen un espacio clave, donde niñas y niños además de aumentar su potencial de aprendizaje, puedan implementar intervenciones de promoción de actividad física y de salud, con el fin de poder desarrollar una mejor calidad de vida. 48

La educadora de párvulo y posteriormente los profesores de educación física son los profesionales quienes conviven diariamente con los infantes en su periodo de escolarización. En ese sentido, tienen un rol preponderante como modelos capaces de influir de forma positiva en el desarrollo de las habilidades motoras, preparando a los niños y niñas para una participación plena, acorde a sus posibilidades y características personales. 49

Brown et al., demostraron que la observación directa a niños por su profesora, aumentaba la intensidad de la actividad física de moderada a vigorosa cuando se comparaba con días no personalizados, dejando claro el rol del docente en la participación de los niños en clases.50 Por otro lado, se ha visto que estrategias de bajo costo implementadas tanto en jardines como colegios, pueden influenciar grandes cambios en los niveles de actividad física de los niños.51,52

En la edad preescolar, una alimentación sana y la promoción del juego, se consideran claves para la formación de buenos hábitos alimentarios y un correcto desarrollo, respectivamente.53 Desde temprana edad es importante entregar estímulos que permitan un adecuado desarrollo motor. Es aconsejable que estas actividades se ejecuten esencialmente, a través de juegos y con una intensidad de moderada a vigorosa, pues se ha visto que están asociadas a la prevención de obesidad y una adecuada condición física.54

La OMS, recomienda a los niños de 5 a 17 años, realizar diariamente 60 minutos de actividad física, los cuales se pueden repartir en varias sesiones a lo largo del día. Además, sugiere adicionar al menos tres veces a la semana ejercicios de resistencia para mejorar la fuerza muscular en los grandes músculos del tronco y las extremidades y ejercicios aeróbicos vigorosos para beneficiar las funciones cardiorrespiratorios y disminuir los factores de riesgo cardiovascular. 21

No se encontraron estudios que permitan esclarecer el protagonismo que representa el sobrepeso/obesidad en el correcto desarrollo motor por sobre otras variables. La presencia y/o combinación de factores, tanto biológicos como socio-culturales, pueden influir con igual o mayor intensidad en la generación de déficits psicomotores. Variables como: la escasa práctica de los patrones motores básicos durante el desarrollo, baja motivación por realizar actividad física, el tipo de actividad física realizada, las barreras percibidas para realizar ejercicio y el tiempo dedicado a actividades de tipo sedentaria pudieran ser estudiadas. Se sugiere investigar en niños normopeso y sobrepeso/obesos diversas condiciones de ejecuciones motrices. Un niño sedentario que no es sobrepeso/obeso podría presentar los mismos resultados que niños sobrepeso/obeso que tienen una gran cantidad de propuestas motrices, de esta forma poder 
esclarecer el protagonismo de las variables que van a desencadenar alteraciones en el desarrollo motor.

Futuras investigaciones deberían profundizar en variables que impactan directamente en la generación del desarrollo motor y las causas de la malnutrición por exceso en los niños, algunas líneas de investigación podrían ser:

- Profundizar en lo que se sabe sobre la diferencias de desarrollo motor entre sexos.

- Estudiar el impacto de los actuales estilos de vida (uso y horas de pantalla en el desarrollo motor y diagnóstico nutricional) en el desarrollo motor de los niños.

- Analizar el impacto de los jardines infantiles, primeros años en la escuela (infraestructura destinada para la actividad física, materiales, tipo, dimensiones de sala) en el desarrollo motor de los niños.

- Estudiar sobre los tipos de ejercicio, cantidad, duración e intensidad óptimos para potenciar el desarrollo motor y prevenir diagnósticos de obesidad y sobrepeso.

- Estudiar el impacto del docente (estilo de clases, porcentaje de tiempo dedicado a la actividad física, nivel de intensidad de las clases, horas de recreo, cantidad de actividad física realizada por los niños en horario de clases) en el desarrollo motor y diagnóstico nutricional de los niños.

- Analizar los efectos de los estilos de vida de los padres (activos, sedentarios) en el desarrollo motor y diagnóstico nutricional de los niños.

En conclusión, si bien los diagnósticos nutricionales de sobrepeso y la obesidad en niños y niñas se asocian más a una disminución en el desarrollo motor en comparación a niños normopesos. Se necesita una mayor cantidad de estudios sobre desarrollo motor y obesidad para aclarar la relación existente entre estas dos variables. Una idea más clara de la relación y posibles efectos del sedentarismo y obesidad infantil en el desarrollo psicomotor infantil nos permitirá tener una visión más integradora de todos los factores que pueden afectar al desarrollo psicomotriz normal de niños y niñas y así poder tomar medidas y estrategias adecuadas de prevención, control o intervención en los plazos más eficaces para que todos los niños tengan la posibilidad de mejorar sus habilidades y rendimiento psicomotor.

Agradecimientos: El autor Igor Cigarroa Cuevas agradece a la Universidad Santo Tomás de Chile por el apoyo a través de la beca de perfeccionamiento académico para realizar este artículo. Además, a CONYCYT/BECA CHILE/PAI 72150035 por su apoyo en el trabajo.

Conflicto de intereses: Ninguno declarado por los autores.

\section{Referencias}

1. Elena A. El aprendizaje motriz en los primeros tres años de vida del niño. Pensam. Educ. 2006;38:218-30.

2. Bucco L, Zubiaur M. Desarrollo de las habilidades motoras fundamentales en función del sexo y del índice de masa corporal en escolares. Cuad. Psicol. del Deport. 2013;13(2):63-72.

3. Moreno F, Ormeño E. Aprendizaje motor y síndrome general de adaptación. Mot. Eur. J. Hum. Movement. 2009;22:1-21.

4. Martínez-López, E, Lara, A, Chacón, J Y Rodríguez I. Characteristics, frequencies and type of physical exercise practiced by the adolescents. Special attention to the obese pupil. J Sport Heal. Res. 2009;1(2):88-100.

5. Bucco L, Zubiaur M. Comparación de la evaluación y desempeño entre la edad cronológica y edad motora general de escolares con medidas corporales de obesidad y sobrepeso. Rev. Digit. 2012;17(175):1.

6. Rodriguez-R A. La obesidad infantil y los efectos de los medios electrónicos de comunicación. Medigraphic Artemisa en linea. 2006;8:95-8.

7. Meitland C, Stratton G, Foster S, Braham R, Rosemberg M. A place for play? The influence of the home physical environment on children's physical activity and sedentary behaviour. Nutr. Phys. Act. 2013;10:99.

8. Crovetto M, Zamorano N, Medinelli A. Estado nutricional, conocimientos y conductas en escolares de kinder y primer año básico en 3 escuelas focalizadas por obesidad infantil en la comuna de Valparaiso, Chile. Rev. Chil. Nutr. 2010;37:309-20.

9. Ray M, Jat K. Effect of electronic media on children. Indian Pediatr. 2010;47(7):561-8.

10. Olivares S, Bustos N, Moreno X, Lera L, Cortes S. Actitudes y prácticas sobre alimentación y actividad física en niños obesos y sus madres en Santiago, Chile. Rev. Chil. Nutr. 2006;33:170-9.

11. Polleti 0, Barrios L. Sobrepeso, obesidad, hábitos alimentarios, actividad física y uso de tiempo libre en 
escolares de corrientes (Argentina). Rev. Cubana Pediatr. 2007;79(1):218-230

12. Guimarães LV, Barros MBA, Martins MSAS, Duarte EC. Fatores associados ao sobrepeso em escolares Revista de Nutrição. 2006; 19(1): 5-17.

13. Suarez P, Hernandez A. Aprendizaje motor: Una breve revisión teórica. efdeportes. 2007;12(109):1.

14. Ruiz G. Hábitos de la práctica lúdica y deportiva en niños y niñas en edad escolar: Un estudio Transversal. Rev. Digit. Educ. Fis. 2011;10:1-15.

15. Kain J, Concha F, Salazar G, Leyton B, Rodriguez M, Ceballos $\mathrm{X}$, et al., Prevención de obesidad en preescolares y escolares de escuelas Municipales de una comuna de Santiago de Chile: Proyecto piloto 2006. Arch. Latinoam. Nutr. 2009;59(2):139-46.

16. Rojas V. Influencia de la televisión y videojuegos en el aprendizaje y conducta infanto-juvenil. 2008;79(1):8085.

17. Chaddock L, Pontifex M, Hillman C, Kramer A. A review of the relation of aerobic fitness and physical activity to brain structure and function in children. J. Int. Neuropsychol. Soc. 2011;17(6):975-85.

18. Singh A, Uijtdewilligen L, Twisk J, Mechelen W, Chinapaw M. Physical activity and performance at school: A systematic review of the literature including a methodological quality assessment. Arch. Pediatr. Adolesc. Med. 2011;166(1):49-55.

19. Thomas-Juste F, Hiromi O, Stafford F. University of Michigan, Institute for Social Research. Changing times of american youth: 1981-2003. Michigan; 2004: 1-15.

20. Khan N, Hillman C. The relation of childhood physical activity and aerobic fitness to brain function and cognition: A review. Pediatr. Exerc. Sci. 2014;26:138146.

21. Organización Mundial de la Salud. Recomendaciones mundiales sobre actividad física para la salud. OMS, 2010. p. 1-57. Disponible en: http://whqlibdoc.who.int/publications/2010/978924 3599977_spa.pdf

22. Chaptup J, Leduc G, Boyer C, Bellanger P, Leblanc A, Borghese $M$, et al. Electronic screens in children's bedrooms and adiposity, physical activity and sleep: do number and type of electronic devices matter? can. J Public Heal. 2014;105:273-9.

23. Seng Y. Consequences of childhood obesity. 2009;38(1):75-81.

24. Haug E, Rasmussen M, Samdal O, Iannotti R, Kelly C, Borraccino A, et al. Overweight in school-aged children and its relationship with demographic and lifestyle factors: Results from the WHO-Collaborative Health Behaviour in School-aged Children (HBSC) Study.Int J Public Health. 2009;2:169-79.

25. Vanden M, Robbins L, Pfeiffer K, Kasanis A, Maier K. Demographic, cognitive, affective, and behavioral variables associated with overweight and obesity in low-active girls. J. Pediatr. Nurs. Elsevier Inc. 2014;29(6):576-85.

26. Kain J, Vio F, Olivares S, Concha F, Uauy R. Prevención de la obesidad infantil: Guía para el desarrollo de intervenciones en alimentación, nutrición y actividad física en escuelas básicas de Chile. 2004:1-20.
27. Ebbeling C, Pawlak D, Ludwig D. Childhood obesity: public-health crisis, common sense cure. Lancet. 2002;360(9331):473-82.

28. Ministerio de Salud Chile. Diagnóstico del estado nutricional de menores de 6 años, gestantes, nodrizas y adultos mayores, bajo control en el sistema publico de salud. Santiago MINSAL. diciembre 2013. Disponible en: http://web.minsal.cl/sites/default/files/DIAGNOSTICO _ESTADO_NUTRICIONAL_DICIEMBRE_2013.pdf

29. Ministerio de Salud Chile. Objetivos sanitarios 20112020: Estrategia nacional de salud para el cumplimiento de los objetivos sanitarios de la década 2011-2020 metas 2011 - 2020. Santiago, MINSAL. Disponible en: http://web.minsal.cl/portal/url/item/c4034eddbc96c a6de0400101640159b8.pdf

30. Ministerio de Salud Chile. Pontificia Universidad Católica de Chile. Universidad Alberto Hurtado. Encuesta Nacional de Salud 2009-2010. Santiago. Disponible en: http://web.minsal.cl/portal/url/item/bcb03d7bc28b6 4dfe040010165012d23pdf

31. OECD Health at a Glance 2011: OECD Indicators, OECD Publishing. Disponible en: http://www.oecd.org/edu/education-at-a-glance19991487.htm

32. Maceira C, Scotto M, Leal M, Lavanda I, Carrazana C, Herrera J, et al.,. Evaluación del estado Nutricional de los niños que participaron en el programa "Activarse" durante el 2011. Act. nutr. 2013;14(1):43-53.

33. Friedemann C, Heneghan C, Mathani K, Thompson M, Perera R, Ward A. Cardiovascular disease risk in healthy children and its association with body mass index: Systematic review and meta-analysis. BMJ. 2012; 345:e4759:1-16.

34. 34 Carrascosa A, Fernández JM, Fernández C, et al. Estudio transversal español de crecimiento 2008. parte II: valores de talla, peso, índice de masa corporal desde el nacimiento hasta la edad adulta. An Pediatr 2008; 68:552-69.

35. CDC. National Center for Health Statistics 2000. Disponible en: http://www.cdc./growthcharts/zscore

36. Reilly J, Kelly J. Long-term impact of overweight and obesity in childhood and adolescence on morbidity and premature mortality in adulthood: systematic review. Int. J. obes (lond). 2011; 35(7):891-8.

37. Gonzalez C, Worley A, Grimmer K, Dones V. Schoolbased interventions on childhood obesity: a metaanalysis. Am. J. Prev. Med. 2009;37(5):418-27.

38. Kain J, Lera L, Rojas J, Uauy R. Obesidad en preescolares de la Región Metropolitana de Chile. Rev. médica Chile. 2007;135(1):63-70.

39. Buring K, Oliva P, Bravo C. Determinación no experimental de la conducta sedentaria en preescolares. Rev. Chil. Nutr. 2009;36(1):23-30.

40. Ostbye T, Krause K, Stroo M, Lovelady C, Evenson K, Peterson B et al. Parent-focused change to prevent obesity in preschoolers: results from the KAN-DO study. Prev. Med. 2012;55(3):188-95.

41. Timmons B, LeBlanc A, Carson V, Connor S, Dillman C, Janssen I et al. Systematic review of physical activity and 
health in the early years (aged 0-4 years). Appl Physiol Nutr Metab. 2012;37:773-92.

42. Carrasco F. Actividad física y obesidad. Medwave. 2002;2(5):3-14.

43. Slining M, Adair L, Davis B, Borja J, Bentley M. Infant overweight is associated with delayed motor development. J. Pediatr. 2010;157(1):20-5.

44. Bustamante A, Caballero L, Enciso N, Salazar I, Teixeira A, Garganta R et al. Coordinación motora: Influencia de la edad, sexo, estatus socio-economico y niveles de adiposidad en niños Peruanos. Rev. Bras. Cineantropom. Desempenho hum. 2008;10(1):25-34.

45. Cano M, Oyarzún T, Leyton F, Sepúlveda C. Relación entre estado nutricional, nivel de actividad física y desarrollo piscomotor. Nutr Hosp. 2014;30(6):1313-18.

46. Mendez M, Estay J, Calzadilla A, Duran S, Díaz-Narváez P. Comparación del desarrollo psicomotor en preescolares chilenos normopeso versus sobrepeso/obesidad. Nutr Hosp. 2015; 32 (1).

47. Roldan E, Paz A. Relación de sobrepeso y obesidad con nivel de actividad física, condición física, perfil psicomotor y rendimiento escolar en población infantil (8 a 12 años) de Popayán. Mov.cient. 2013; 7 (1): 71-84.

48. Ibarra J, Alarcon M. Malnutrition by Excess among School- Age Children. Rev Chil Pediatr. 2010;81(6):50614.

49. Ministerio de Educación. Bases curriculares de la educación parvularia. Santiago: Nuestra riqueza. MINEDUC. 2001.

50. Brown W, HS, Smith H, McIver K, Rathel J. Effects of teacher-encouraged physical activity on preschool playgrounds. J Early Interv.2013;31(2):126-45.

51. Cardon G, Labarque V, Smits D, Bourdeaudhuij I. Promoting physical activity at the pre-school playground: The effects of providing marking and play equipment. Prev. Med. 2009;48:335-40.

52. Willenberg L, Ashbolt R, Holland D, Gibbs L, Macdougall $\mathrm{C}$, Garrard J et al. Increasing school playground physical activity: A mixed methods study combining environmental and children's perspectives. J. Sci. Med. Sport Meas. J. Sci. 2010;13:210-16.

53. Goldfield G, Harvey A, Grattan K, Adamo K. Physical activity promotion in the preschool years: a critical period to intervene. Int. J. Environ. Res. Public Health. 2012;9(4):1326-42.

54. Fisher A, Reilly J, Kelly L, Montgomery C, Williamson A, Paton J et al. Fundamental movement skills and habitual physical activity in young children. Med. Sci. Sport. Exerc. 2005;37(4):684-8. 